\title{
Stereotactic fractionated irradiation of optic nerve sheath meningioma: a new treatment alternative
}

\author{
S Pitz, G Becker, U Schiefer, H Wilhelm, B Jeremic, M Bamberg, E Zrenner
}

Br J Ophthalmol 2002;86:1265-1268

See end of article for authors' affiliations

Correspondence to: Susanne Pitz, MD, University Eye Hospital, Langenbeckstrasse 1, D 55101 Mainz, Germany; pitz@augen.klinik.

uni-mainz.de

Accepted for publication 2 July 2002
Background: Primary optic nerve sheath meningioma (ONSM) is a rare but almost invariably blinding tumour when its natural history is observed in a "wait and see" strategy. Surgery has hitherto only been advocated in case of progressive disease involving intracranial structures, as it leads to iatrogenic blindness in the overwhelming majority of cases. Therefore, treatment options bearing lesser risk of functional deterioration are highly desirable, both in cases of intracranial involvement as well as during earlier phases of the disease which are currently generally left untreated. The authors report the outcome of the largest series of patients to date treated by stereotactic fractionated irradiation as a new treatment approach in ONSM at all stages.

Methods: 15 patients (16 nerves) underwent stereotactic fractionated conformal irradiation with a total dose of $54 \mathrm{~Gy}$, using standard fractionation. Main outcome parameters included visual acuity and visual field, as well as three dimensional remission as documented by imaging.

Results: Tumour control was confirmed in all 15 patients undergoing stereotactic fractionated conformal irradiation (mean follow up 37 (range 12-71) months). No patient developed functional deterioration during or after treatment. Moreover, visual acuity improved by more than two lines in one patient and the visual field improved in six cases. Visual outcome in the other patients remained unchanged. There were no significant side effects of radiation therapy.

Conclusion: These data provide convincing evidence that stereotactic fractionated conformal irradiation is an effective treatment option for primary ONSM with minimal treatment related morbidity. It should therefore be considered as therapeutic option both in early stage ONSM where surgery cannot be justified as well as in later stages, where surgery is so far considered the first line approach.
$\mathrm{P}$ rimary optic nerve sheath meningiomas (ONSM) arise from the intraorbital and/or intracanalicular arachnoidal sheath of the optic nerve. They usually occur unilaterally, and represent approximately $96 \%$ of all orbital and $1-2 \%$ of all intracranial meningiomas. ${ }^{1}$ Though prognosis for life is excellent, these tumours almost always lead to complete visual loss in the affected eye. Tumour progression, however, may be exceedingly slow or even absent over extended periods of time. This is of particular importance in view of the invariable deterioration of visual function after surgical intervention, which may prevent disfiguring exophthalmos or complications of intracranial involvement, but not visual loss. ${ }^{1}$ Therefore, alternative treatment options providing a better functional outcome are highly desirable.

Since the early 1980s a number of promising reports on the use of conventional radiotherapy in the treatment of ONSM have been published..$^{2-6}$ Conformal stereotactic fractionated irradiation protocols have evolved in the more recent past, giving evidence that this treatment may permanently preserve or even restore vision with a minimum of adverse reactions; however, the total number of patients reported to have been treated by this new approach is small. ${ }^{6-10}$ We report on 15 consecutive patients with primary ONSM treated according to a standardised stereotactic fractionated irradiation protocol.

\section{PATIENTS AND METHODS}

Between 1989 and 2000, 15 patients (12 females, mean age 51.5 years; three males; mean age 35 years) were seen at the University Eye Hospital Tübingen for primary ONSM with signs of progressive disease (Table 1). Careful medical and neurological evaluation was performed in each patient to exclude systemic disease. Confirmatory biopsy was carried out in three patients. In all others, diagnosis was based on clinical criteria and the typical radiographic appearance on magnetic resonance imaging (MRI) or computed tomography (CT).

Ophthalmological investigation included best corrected visual acuity tested with a Snellen chart ${ }^{11}$ at a 5 metre distance, assessment of a relative afferent pupillary defect (RAPD), motility testing, slit lamp investigation of the anterior segment, and funduscopy. Testing of visual fields was done with the Goldmann perimeter, and within the $30^{\circ}$ area with the Tübingen manual perimeter or Tübingen automated perimeter, respectively. To obtain reliable results on follow up, only similar performance measurements recorded were considered, and only absolute scotomas were evaluated. The evaluation of visual fields was done using a modified Esterman grid, ${ }^{12}$ indicating the percentage of visual field loss by counting the number of absolute defects.

Criteria for relevant deterioration or amelioration were as follows: decline or increase of visual acuity of two or more lines, change of at least $8 \%$ of visual field, or progression in imaging.

In the presence of suspected deterioration, ophthalmological evaluation was done at 3-6 month intervals, and MRI scans were repeated at 6 month intervals. In patients with stable disease, clinical evaluation and imaging were performed on an annual basis. In patients treated with radiotherapy, follow up examinations (ophthalmological, radio-oncological, endocrinological, and by MRI) were done at 3 month intervals for the first year after therapy. During the second year of follow up, ophthalmological and radiooncological evaluations were carried out at 6 month intervals, while endocrinological examinations and MRI were performed once a year. As of the third year of follow up, control examinations were performed on an annual basis.

Radiation therapy consisted of $6 \mathrm{MV}$ photons from linear accelerators. The radiotherapy treatment regimen consisted of 
Table 1 Patient characteristics and initial visual function

\begin{tabular}{|c|c|c|c|c|c|c|c|c|}
\hline \multirow[b]{2}{*}{ Patient No } & \multirow{2}{*}{$\begin{array}{l}\text { Age at diagnosis } \\
\text { (years) }\end{array}$} & \multirow{2}{*}{$\begin{array}{l}\text { Symptoms } \\
\text { before diagnosis } \\
\text { (months) }\end{array}$} & \multirow{2}{*}{ Sex } & \multirow{2}{*}{$\begin{array}{l}\text { Affected } \\
\text { side }^{*}\end{array}$} & \multirow[b]{2}{*}{ Location† } & \multicolumn{2}{|c|}{ Initial examination } & \multirow{2}{*}{$\begin{array}{l}\text { Follow up } \\
\text { before } \\
\text { treatment } \\
\text { (months) }\end{array}$} \\
\hline & & & & & & Visual acuity $\ddagger$ & Visual field loss & \\
\hline 1 & 64 & 11 & $\mathrm{~F}$ & I & 1 & $0.4 \S$ & $70 \%$ & 5 \\
\hline 2 || 9 & 31 & 37 & $\mathrm{~F}$ & $b / l$ & 4 & 1.0 & $34 \%$ & 7 \\
\hline 3 & 67 & 10 & $\mathrm{~F}$ & r & 2 & 1.0 & $70 \%$ & 13 \\
\hline 4 & 58 & 81 & $\mathrm{~F}$ & $r$ & 3 & 1.0 & $6 \%$ & 2 \\
\hline 59 & 42 & 70 & $\mathrm{~F}$ & I & 1 & 0.8 & $5 \%$ & 21 \\
\hline $6 \pi^{* *}$ & 35 & 36 & M & $b$ & 2 & 1.0 & $84 \%$ & 4 \\
\hline $7 \mathbb{1}^{* *}$ & 13 & 24 & $M$ & $r$ & 1 & FC & $100 \%$ & 5 \\
\hline 8 & 57 & 5 & $\mathrm{~F}$ & $r$ & 1 & 1.0 & $0 \%$ & 35 \\
\hline 9 & 44 & 12 & $\mathrm{~F}$ & 1 & 2 & $0.63 \uparrow \dagger$ & $0 \%$ & 99 \\
\hline 10 & 53 & 5 & $\mathrm{~F}$ & $r$ & 3 & 0.8 & $60 \%$ & 12 \\
\hline 119 & 29 & 61 & $\mathrm{~F}$ & $r$ & 1 & 1.25 & $0 \%$ & 16 \\
\hline 12 ** & 50 & 120 & $F$ & 1 & 2 & 1.0 & $0 \%$ & 4 \\
\hline 13 & 39 & 6 & $M$ & I & 1 & 1.0 & $0 \%$ & 84 \\
\hline 14 & 66 & 9 & $\mathrm{~F}$ & $r$ & 2 & 0.8 & NK & 4 \\
\hline $15 \Phi$ & 38 & 12 & $\mathrm{~F}$ & r & 3 & $\mathrm{LP}$ & NK & 3 \\
\hline 2 || & 31 & 37 & $F$ & $b / r$ & 3 & NLP & $100 \%$ & 7 \\
\hline \multicolumn{9}{|c|}{$\begin{array}{l}\text { * I = left; } r=\text { right; } b=\text { bilateral. } \\
\text { †Location: } 1=\text { orbital; } 2=\text { orbital and optic nerve canal; } 3=\text { orbit, optic nerve canal, and intracranial extension; } 4 \text { = canalicular. } \\
\text { †NLP = no light perception; } L P=\text { light perception; } F C=\text { finger counting. } \\
\text { §Low visual acuity due to inadequate spectacle correction. } \\
\text { \| Patient No } 2 \text { is suffering from bilateral ONSM, therefore mentioned twice. } \\
\text { TAdditional systemic steroidtherapy. } \\
\text { * *Biopsy. } \\
\text { ††Low visual acuity due to amblyopia. }\end{array}$} \\
\hline
\end{tabular}

doses of 50.4 Gy in 26 daily fractions prescribed to an ICRU (International Conference on Radiation Units) reference point with a safety margin of $5 \mathrm{~mm}$; the boost consisted of $3.60 \mathrm{~Gy}$ in two daily fractions with a safety margin of $2 \mathrm{~mm}$. The total prescribed tumour dose was $54 \mathrm{~Gy}$ in 28 fractions over 5.5 weeks. The therapeutic radiation dose was achieved by three to six non-coplanar irregular fields with the head of the patient positioned in an individually designed device allowing exact and reproducible attachment to the stereotactic base frame. The computer assisted three dimensional treatment planning was based on MRI and CT imaging data. Precision was achieved using a stereotactic system designed at the German Cancer Research Centre (DKFZ), Heidelberg, Germany. ${ }^{13}$

\section{RESULTS}

All patients included in this study exhibited signs of tumour progression within a mean observation period of 7 months. Therefore, stereotactic radiation therapy was initiated. At initial investigation visual acuity in 10 of these patients was $>0.8$, a normal visual field ( $<8 \%$ of visual field loss) was present in six; one patient (No 2) with bilateral disease had two unequally affected eyes, with unimpaired vision in one and blindness in the contralateral eye (Table 1).

After a mean follow up period of 37 (range 12-71) months following treatment, none of the seeing eyes treated suffered form deterioration of visual function. An increase in visual acuity of two or more lines was found in one patient; amelioration of visual field defects of at least $8 \%$ was noted in five; patient No 2 experienced a dramatic improvement in her better eye from $72 \%$ visual field loss to $4 \%$ (Table 2 ). Stable disease was observed in eight patients. However, three of these patients were already blind or nearly blind at treatment initiation; in addition, patient No 2 was blind in one affected eye. In these cases, improvement of vision with treatment cannot be expected.

Thus, functional improvement was achieved in seven out of $15(46 \%)$ patients, and seven out of $16(43 \%)$ eyes (including the better eye of patient No 2). Excluding the eyes already blind at treatment initiation, functional improvement was achieved in seven out of $12(58 \%)$ eyes, none deteriorated.

Radiographic (CT and/or MRI) tumour control was achieved in all $(100 \%)$ patients. Acute side effects included local erythema in five and local alopecia in 11 patients. These findings resolved during the follow up period. Endocrinological evaluation revealed functional hyperprolactinaemia in two and partial hypophyseal insufficiency in one patient.

\section{DISCUSSION}

Surgery of primary ONSM leads to severe functional deterioration in about $95 \%$ of patients due to the unavoidable dissection of the pial vascular supply of the optic nerve. ${ }^{15}$ In addition, it bears a considerable risk of incomplete removal because multiple sites or "micrometastases" may be indiscernible during surgery, or tumour cells may be spread despite meticulous technique. ${ }^{1}$ Nevertheless, surgical treatment still is considered the gold standard based on the published clinical data and results of long term follow up studies. ${ }^{15} 16$

However, conventional ${ }^{2-6}$ and, more recently, stereotactic fractionated radiation ${ }^{6-10}$ have emerged as promising treatment alternatives for patients with ONSM. This must not be confused with stereotactic radiosurgery, which has also been used in the treatment of this condition. ${ }^{17}$ Stereotactic radiosurgery-in contrast with stereotactic radiotherapyapplies higher single radiation doses in one or a few boluses. There is thus considerable concern regarding damage to adjacent cerebral structures, the optic nerve, and/or the retina. ${ }^{18}$

Conventional as well as stereotactic radiation therapy has in the past been used primarily as adjunctive therapy in combination with surgery, ${ }^{3419}$ although there are some reports on the application of this approach as the sole intervention..$^{23-9} 20$ Conformal stereotactic fractionated radiation protocols (combining adequate dose delivery to the tumour with minimal possible radiation induced side effects on the surrounding structures) may therefore represent a more adequate approach in the therapy of ONSM.

The most intriguing finding in our series was the improvement of the visual field in six of 15 patients. In two of these patients the visual field was restored to a near normal condition (Nos 1 and 2, Table 2), although before treatment visual field defects of more than $50 \%$ had been documented. The improvement was less marked in the remaining four patients. Interestingly, visual acuity remained unchanged in two of these four patients (Nos 5 and 6, Table 2). This implies that irradiation in a patient with a field defect and concomitant 
Table 2 Treatment data

\begin{tabular}{|c|c|c|c|c|c|c|c|}
\hline \multirow[b]{2}{*}{ Patient No } & \multicolumn{2}{|c|}{ Pretreatment examination } & \multirow[b]{2}{*}{$\begin{array}{l}\text { Progression in } \\
\text { imaging }\end{array}$} & \multicolumn{2}{|c|}{ Most recent examination } & \multirow{2}{*}{$\begin{array}{l}\text { Follow up after } \\
\text { treatment } \\
\text { (months) }\end{array}$} & \multirow[b]{2}{*}{ Outcome } \\
\hline & Visual acuity $\ddagger$ & Visual field loss & & Visual acuity $\ddagger \ddagger$ & $\begin{array}{l}\text { Visual field } \\
\text { loss } \ddagger \ddagger\end{array}$ & & \\
\hline 1 & $0.6 \S$ & $72 \%$ & - & 1.0 & $4 \%$ & 43 & Improved \\
\hline 2 || & 1.0 & $72 \%$ & - & 1.2 & $4 \%$ & & Improved \\
\hline 3 & 1.0 & $92 \%$ & - & 1.0 & $78 \%$ & 46 & Improved \\
\hline 4 & 1.2 & $68 \%$ & + & 1.0 & $40 \%$ & 16 & Improved \\
\hline 59 & 0.8 & $58 \%$ & - & 0.6 & $49 \%$ & 49 & Improved \\
\hline $6 \pi^{* *}$ & 0.4 & $81 \%$ & + & 0.4 & $73 \%$ & 21 & Improved \\
\hline $79^{* *}$ & FC & $84 \%$ & - & 0.1 & NK & 37 & Improved \\
\hline 8 & 1.0 & $0 \%$ & + & 0.8 & $6 \%$ & 12 & Stable \\
\hline 9 & $0.32 \dagger \dagger$ & $66 \%$ & + & 0.2 & $64 \%$ & 22 & Stable \\
\hline 10 & 0.63 & $68 \%$ & - & 0.8 & $72 \%$ & 45 & Stable \\
\hline 119 & 1.25 & $2 \%$ & - & 1.2 & $2 \%$ & 32 & Stable \\
\hline 12 ** & 0.8 & $2 \%$ & + & 0.8 & NK & 23 & Stable \\
\hline 13 & $\mathrm{LP}$ & $100 \%$ & + & $L P$ & $100 \%$ & 15 & Stable/blind \\
\hline 14 & $\mathrm{LP}$ & $100 \%$ & - & NLP & $100 \%$ & 43 & Stable/blind \\
\hline 159 & NLP & $100 \%$ & - & NLP & $100 \%$ & 71 & Stable/blind \\
\hline $2 \|$ | & LP & $100 \%$ & - & NLP & $100 \%$ & 38 & Stable/blind \\
\hline \multicolumn{8}{|c|}{$\begin{array}{l}\text { * }=\text { left; } r=\text { right; } b=\text { bilateral. } \\
\text { †Location: } 1 \text { = orbital; } 2=\text { orbital and optic nerve canal; } 3=\text { orbit, optic ner } \\
\text { †NLP = no light perception; } L P=\text { light perception; } F C=\text { finger counting. } \\
\text { §Low visual acuity due to inadequate spectacle correction. } \\
\| \text { Patient No } 2 \text { is suffering from bilateral ONSM, therefore mentioned twice. } \\
\text { IAdditional systemic steroidtherapy. } \\
\text { **Biopsy. } \\
\text { ††Low visual acuity due to amblyopia. } \\
\pm \pm B o l d \text { figures denote amelioration. }\end{array}$} \\
\hline
\end{tabular}

impairment of visual acuity does not necessarily lead to an improvement of both conditions. This finding is in accordance with results of other case reports on stereotactic fractionated irradiation protocols in ONSM. ${ }^{78}$

An improvement of visual acuity of at least two lines was found in one patient (field measurements for this patient were not available after radiation). This low rate of improved visual acuity is in contrast with results obtained by others. ${ }^{25}{ }^{10}$ These studies reported marked improvements of visual acuity in a significant number of patients. The reason for our results with regard to this parameter may be partly accounted for by the criteria for patient selection: all of Kennerdell's patients had an initial visual acuity ranging from 0.2 to $0.5 .^{5}$ This "mid-range acuity" is under-represented in our patients. Surprisingly, a very recent study of the same group comprising 34 patients (including those patients reported on earlier) undergoing varying irradiation protocols does not confirm these optimistic results of the initial study. ${ }^{6}$ An underestimation of the therapeutic effect might be assumed, owing to the fact that the authors chose to compare visual acuity at the time of diagnosis of the disease rather than at initiation of radiation therapy with the visual outcome. Turbin and coworkers describe a stabilisation, but not improvement of visual acuity. This leads us to conclude that once visual acuity is impaired, it may not be fully restored.

Four of 16 eyes in our study were blind at onset of treatment, while five of 16 eyes had a visual acuity of 1.0 or better. Therefore, improvement of vision could not be achieved because of either excellent or lost baseline vision. Consequently, if radiation therapy is considered, it should be initiated early in the course of the disease.

There are limitations for any study dealing with ONSM. ONSM is a rare disease, and it is difficult even for a specialised referral centre such as ours to accumulate enough patient data to obtain statistically significant observations. The course of the disease is slow, and it would need multicentre studies lasting for decades to develop evidence based recommendations on disease management. At the same time, therapeutic and diagnostic possibilities improve ever more rapidly and those applied at the begin of a study might be inadequate at its end. All current treatment recommendations are therefore based on retrospective studies.
An additional problem is the follow up period. It should be long enough to allow the detection of late recurrence as well as late side effects of irradiation. Our follow up of about 3 years is not long enough to fully exclude either possibility. However, radiation induced side effects after this period are rare. ${ }^{21}$ Sibony and coworkers, evaluating the natural course of ONSM, reported all patients to exhibit a decline in visual acuity in a mean observation period of 2.2 years. ${ }^{22}$

Given these observations and taking into account the 95\% vision deterioration rate following surgery, we nevertheless feel confident to conclude that stereotactic fractionated conformal radiotherapy can stop the progression of ONSM. However, restoration of lost visual function, though definitively possible and realised in about half of our patients, cannot be reliably predicted based on pretreatment characteristics. We therefore recommend consideration of radiotherapy early in the course of the disease as soon it becomes evident that clinically significant visual impairment develops.

\section{Authors' affiliations}

S Pitz, Department of Ophthalmology, Johannes Gutenberg-University, Langenbeckstrasse 1, 55101 Mainz, Germany

S Pitz, U Schiefer, H Wilhelm, E Zrenner, University Eye Hospital, Department of Pathophysiology of Vision and Neuro-Ophthalmology, Schleichstrasse 12-16, 72076 Tübingen, Germany

G Becker, B Jeremic, M Bamberg, Department of Radiooncology, University Hospital, Hoppe-Seyler-Strasse 3, 72076 Tübingen, Germany G Becker, Department of Radiooncology, Klinik am Eichert,

Eichertstrasse 3, 73035 Göppingen, Germany

None of the authors has any financial or proprietary interest in any item described in the presented study nor has there been any private or pubic support.

\section{REFERENCES}

1 Dutton JJ. Optic nerve sheath meningiomas. Surv Ophthalmol 1992; 37: 167-83.

2 Smith JL, Vuksanovic MM, Yates BM, et al. Radiation therapy for primary optic nerve meningiomas. J Clin Neuro-ophthalmol $1981 ; 1: 85-99$

3 Kupersmith MJ, Warren FA, Newall J, et al. Irradiation of meningiomas of the intracranial anterior visual pathway. Ann Neurol 1987;21:131-7.

4 Ito $M$, Ishizawa A, Miyaoka M, et al. Intraorbital meningiomas. Surgical management and role of radiation therapy. Surg Neurol 1988;29:448-53. 
5 Kennerdell JS, Maroon JC, Malton M, et al. The management of optic nerve sheath meningiomas. Am J Ophthalmol 1988;106:450-7.

6 Turbin RE, Thompson CR, Kennerdell JS, et al. A long-term visual outcome comparison in patients with optic nerve sheath meningioma managed with observation, surgery, radiotherapy, or surgery and radiotherapy. Ophthalmology 2002;109:890-9.

7 Eng T, Albright N, Kuwahara G, et al. Precision radiation therapy for optic nerve sheath meningiomas. Int J Radiat Oncol Biol Phys 1992;22:1093-8.

8 Lee AG, Woo SY, Miller NR, et al. Improvement in visual function in an eye with a presumed optic nerve sheath meningioma after treatment with three-dimensional conformal irradiation therapy. J Neuro-Ophthalmol 1996; 16:247-51.

9 Fineman MS, Augsburger JJ. A new approach to an old problem. Surv Ophthalmol 1999;43:519-24.

10 Moyer PD, Golnik KC, Breneman J. Treatment of optic nerve sheath meningioma with threeBdimensional conformal radiation. Am J Ophthalmol 2000:129:694-6.

11 Duke-Elder S, Smith RJH. The examination of the visual functions. In: Duke-Elder S, ed. The foundations of ophthalmology: hereditary, pathology, diagnosis, and therapeutics. System of ophthalmology. Vol 7 . St Louis: CVG Mosby, 1962:370-4.

12 Esterman B. Grid for scoring visual fields. Arch Ophthalmol 1968;79:400-6.
13 Becker G, Schlegel W, Major J, et al. Stereotactic convergent beam radiosurgery versus stereotactic conformation beam radiotherapy. Acto Neurochirurg 1995;63:44-51

14 Becker G, Kortmann RD, Duffner F, et al. High precision external radiotherapy of brain tumours. Onkologie 1998;21:290-7.

15 Wright JE, McNab AA, McDonald WI. Primary optic nerve sheath meningioma. Br J Ophthalmol 1989;73:960-6.

16 Jakobiec FA, Jones IS. Neurogenic tumors. In: Tasman W, Jaeger EA, eds. Duane's clinical ophthalmology. Philadelphia: Lippincott, 1995:Vol 2 , chapter 41

17 Klink DF, Miller NR, Williams J. Preservation of residual vision 2 years after stereotactic radiosurgery for a presumed optic nerve sheath meningioma. J Neuro-Ophthalmol 1998;18:117-20.

18 Kondziolka D, Niranjan A, Lunsford LD, et al. Stereotactic radiosurgery for meningiomas. Neurosurg Clin N Am 1999;10:317-25

19 Peele KA, Kenerdell JS, Maroon JC, et al. The role of postoperative irradiation in the management of sphenoid wing meningiomas. Ophthalmology 1996:103:1761-7.

20 Grant III W, Cain RB. Intensity modulated conformal therapy for intracranial lesions. Medical Dosimetry 1998;23:237-41

21 Capo H, Kupersmith M. Efficacy and complications of radiotherapy of anterior visual pathway fumors. Neuro-ophthalmology 1991;9:179-203.

22 Sibony PA, Krauss HR, Kennerdell JS, et al. Optic nerve sheath meningiomas. Clinical manifestations. Ophthalmology $1984 ; 91: 1313-26$

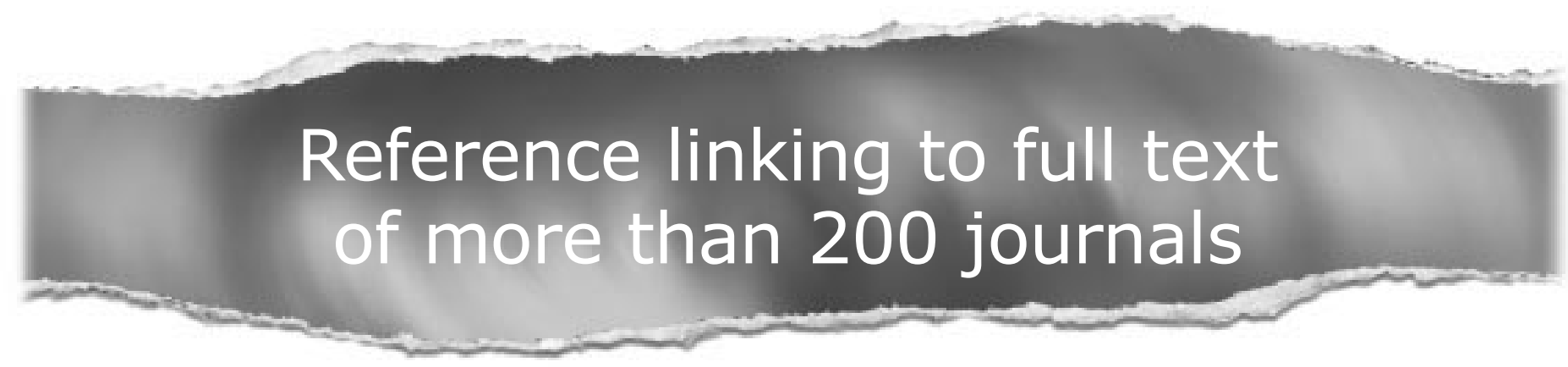

\section{Toll free links}

You can access the FULL TEXT of articles cited in the British Journal of Ophthalmology online if the citation is to one of the more than 200 journals hosted by HighWire (http://highwire.stanford.edu) without a subscription to that journal. There are also direct links from references to the Medline abstract for other titles.

www.bjophthalmol.com 